\title{
Netrin-1 Promotes Thalamic Axon Growth and Is Required for Proper Development of the Thalamocortical Projection
}

\author{
Janet E. Braisted, ${ }^{1}$ Susan M. Catalano, ${ }^{2}$ Robert Stimac, ${ }^{1}$ Timothy E. Kennedy, ${ }^{3}$ Marc Tessier-Lavigne, ${ }^{3}$ \\ Carla J. Shatz, ${ }^{2}$ and Dennis D. M. O'Leary ${ }^{1}$ \\ ${ }^{1}$ Molecular Neurobiology Laboratory, The Salk Institute, La Jolla, California 92037, 2Department of Molecular and Cell \\ Biology, University of California, Berkeley, California 94720, and ${ }^{3}$ Departments of Anatomy and of Biochemistry and \\ Biophysics, University of California, San Francisco, California 94143
}

The thalamocortical axon (TCA) projection originates in dorsal thalamus, conveys sensory input to the neocortex, and has a critical role in cortical development. We show that the secreted axon guidance molecule netrin-1 acts in vitro as an attractant and growth promoter for dorsal thalamic axons and is required for the proper development of the TCA projection in vivo. As TCAs approach the hypothalamus, they turn laterally into the ventral telencephalon and extend toward the cortex through a population of netrin-1-expressing cells. DCC and neogenin, receptors implicated in mediating the attractant effects of netrin-1, are expressed in dorsal thalamus, whereas unc5h2 and unc5h3, netrin-1 receptors implicated in repulsion, are not. In vitro, dorsal thalamic axons show biased growth toward a source of netrin-1, which can be abolished by netrin-1-blocking antibodies. Netrin-1 also enhances overall axon outgrowth from explants of dorsal thalamus. The biased growth of dorsal thalamic axons toward the internal capsule zone of ventral telencephalic explants is attenuated, but not significantly, by netrin-1-blocking antibodies, suggesting that it releases another attractant activity for TCAs in addition to netrin-1. Analyses of netrin-1 -/- mice reveal that the TCA projection through the ventral telencephalon is disorganized, their pathway is abnormally restricted, and fewer dorsal thalamic axons reach cortex. These findings demonstrate that netrin-1 promotes the growth of TCAs through the ventral telencephalon and cooperates with other guidance cues to control their pathfinding from dorsal thalamus to cortex.

Key words: axon guidance; chemoattraction; DCC; dorsal thalamus; internal capsule; neocortex; neogenin; striatum; Unc5 homologs
One of the major axon tracts in the mammalian forebrain is the internal capsule (IC), which is the path of cortical efferent axons and thalamocortical axons (TCAs) through the ventral telencephalon. TCAs originate in dorsal thalamus and relay sensory information from the periphery to the neocortex (Jones, 1985). In addition, TCAs are required for the proper differentiation of cortical layers and areas (Chenn et al., 1997). During development, TCAs extend ventrally from dorsal thalamus along the lateral surface of ventral thalamus. As TCAs approach the hypothalamus, they make a sharp lateral turn to enter the ventral telencephalon at a specific location and extend dorsolaterally toward the neocortex along a path, which here we term the internal capsule zone (ICZ) (Fig. 1) (Braisted et al., 1999; Tuttle et al., 1999).

Although the mechanisms directing the pathfinding of TCAs to the neocortex are not well understood, recent studies suggest that a variety of spatially and functionally distinct cues are involved. Contact-mediated cues have been implicated in the guidance of TCAs into and through the ICZ. Although the details differ between them, axon tracing studies in hamster (Metin and Godement, 1996), mouse (Braisted et al., 1999; Tuttle et al., 1999), and rat (Molnar et al., 1998) suggest that TCAs use the axons of neurons situated in the ICZ that project into dorsal thalamus as a scaffold to enter the ICZ. Supporting this hypothesis, in mice deficient for the basic helix-loop-helix transcription factor gene

Received Dec. 17, 1999; revised May 11, 2000; accepted May 12, 2000.

This work was supported by National Institutes of Health Grants R01 NS31558 (D.D.M.O'L.) and R01 EY02858 (C.J.S.), and fellowship National Research Service Award EY06491 (S.M.C.). C.J.S. and M.T.L. are Investigators of the Howard Hughes Medical Institute.

Correspondence should be addressed to Dennis D. M. O'Leary, MNL-O, The Salk Institute, 10010 North Torrey Pines Road, La Jolla, CA 92037. E-mail: doleary@salk.edu.

Dr. Kennedy's present address: Montreal Neurological Institute, Montreal, Quebec Canada H3A 2B4.

Dr. Catalano's present address: Roche Bioscience, R2-101, 3401 Hillview Avenue, Palo Alto, CA 94304.

Copyright (C) 2000 Society for Neuroscience $0270-6474 / 00 / 205792-10 \$ 15.00 / 0$
Mash-1, the population of ICZ cells identified to project early on to dorsal thalamus is absent, and TCAs fail to turn and extend into the ICZ (Tuttle et al., 1999). In addition, cortical subplate axons have been suggested to provide a scaffold for the pathfinding of TCAs through the distal portion of the ICZ and to their area-specific targets in the neocortex (Molnar et al., 1998). Molecules secreted by tissues lying along the path of TCAs also appear to be involved in their guidance. Coculture studies suggest that a chemorepellent released by the hypothalamus and a chemoattractant released by the ICZ are involved in directing the pathfinding of TCAs (Braisted et al., 1999). However, the molecular identities of these factors remain to be determined.

The axon chemoattractant netrin-1 (Kennedy et al., 1994; Serafini et al., 1994) is expressed in the ventral telencephalon at the time TCAs navigate through the ICZ (Serafini et al., 1996; Metin et al., 1997). The principle goals of this study were to determine whether netrin-1 is an ICZ attractant activity for TCAs and whether it is required for TCA pathfinding. To achieve these goals, we have determined the relevant expression patterns of netrin-1 and its receptors, have used in vitro axon guidance assays for the action of netrin-1 on dorsal thalamic axons, and have analyzed the pathfinding of TCAs in mice deficient for netrin-1. Our findings implicate netrin- 1 as an attractant and growth promoter for TCAs and show that it is required for the proper development of the TCA projection.

\section{MATERIALS AND METHODS}

Animals. ICR mice from embryonic day 13.5 (E13.5)-E14.5 were obtained from timed-pregnant females (Harlan Sprague Dawley, Indianapolis, IN). Embryos were staged according to Butler and Juurlink (1987). The day of insemination was designated as E0. Production and breeding of netrin-1deficient mice was as previously described (Skarnes et al., 1995; Serafini et al., 1996). Genotyping of E15 to postnatal day 0 (P0) embryos was performed by X-gal staining and verified using morphological criteria; the pons, corpus callosum, anterior, and hippocampal commissures are absent in homozygous netrin-1 mutant embryos $(-/-)$, but present in heterozygous $(+/-)$ and wild type netrin-1 $(+/+)$ embryos (Serafini et al., 1996). For X-gal staining, brains were dissected free, and the remaining tissues 


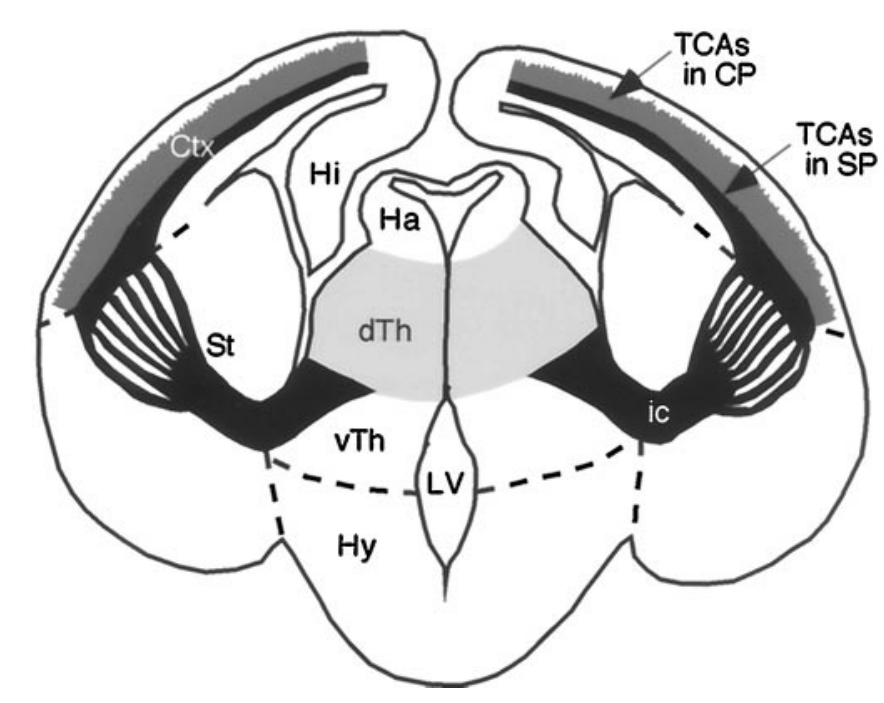

Figure 1. TCA pathway. Schematic of a coronal section of mouse brain showing the mature TCA projection and relevant brain structures. TCAs (black), originating in dorsal thalamus ( $d T h$; light gray), project ventrally along the lateral aspect of ventral thalamus $(v T h)$. As they approach the hypothalamus $(H y)$, TCAs make a sharp lateral turn and extend dorsolaterally through the striatum $(S t)$ toward neocortex $(C t x)$. TCAs are compactly bundled along the proximal portion of their path, but fan-out into numerous fascicles as they extend dorsolaterally through the striatum. As TCAs extend through the Ctx, their path becomes centered on the subplate layer $(S P)$, and branches of TCAs (medium gray) extend into and innervate the cortical plate $(C P)$. TCAs, together with the oppositely extending corticothalamic axons, form the internal capsule (ic), the major axon tract for cortical input and output projections. Dashed lines indicate the approximate borders between the ventral thalamus and hypothalamus, diencephalon and striatum, and striatum and neocortex.

were histochemically processed in a solution containing $1 \mathrm{mg} / \mathrm{ml} \mathrm{X-gal,} 5$ $\mathrm{mm} \mathrm{K}$ ferricyanide, $5 \mathrm{~mm} \mathrm{~K}$ ferrocyanide, $2 \mathrm{~mm} \mathrm{MgCl} 2,0.01 \% \mathrm{Na}$ deoxycholate, and $0.02 \%$ NP-40 in PBS. Within an hr, the netrin-1 - / - embryos could be readily distinguished from the netrin $-1+/-$ and $+/+$ embryos. The $-/-$ embryos have twice the X-gal staining intensity as the $+/-$ embryos, whereas the $+/+$ embryos have no X-gal staining.

In situ hybridization. In situ hybridizations of $20 \mu \mathrm{m}$ cryosections were performed as previously described (Tuttle et al., 1999). Digoxigeninlabeled cRNA probes to netrin-1, DCC, neogenin, unc 5h2, and unc 5 h 3 were synthesized from cDNAs as described by Friedman and O'Leary (1996). Probes were synthesized from a $2.7 \mathrm{~kb}$ fragment of netrin- 1 cDNA (encoding the $3^{\prime}$ UTR region lacking the poly-A tail), an $850 \mathrm{bp}$ fragment of DCC cDNA (encoding bp $3786-4639$ of the cytoplasmic region), an $800 \mathrm{bp}$ fragment of neogenin cDNA (encoding bp 1117-1922 including the fourth Ig domain to third FN III repeat), a $1.5 \mathrm{~kb}$ domain of $u n c 5 h 2 \mathrm{cDNA}$, and a $1.6 \mathrm{~kb}$ fragment of unc $5 \mathrm{~h} 3 \mathrm{cDNA}$.

Preparation of explants. Embryos were removed from anesthetized timed-pregnant mice (E13.5-E14.5) and rats (E17-E18). To isolate the ICZ and dorsal thalamus, embryonic mouse brains were removed, embedded in 3\% low melting point agar (Seaplaque FMC Bioproducts) in L-15 medium supplemented with $0.6 \%$ glucose (Sigma, St. Louis, MO; L15glucose), and sectioned coronally at $200 \mu \mathrm{m}$ using a vibratome. Sections where the IC bridges the diencephalon and ICZ were collected, and the neocortex and diencephalon were removed. This piece served as the ICZ explant (Fig. $2 A$, boxed area). Dorsal thalamus was isolated from the remaining diencephalic pieces, as well as from more caudal sections, by making two cuts-one through the external medullary lamina/medial lemniscus to remove the ventral thalamus and hypothalamus and a second dorsal cut to remove the epithalamus. The resulting piece of tissue was bisected to yield two explants-one from lateral dorsal thalamus and one from medial dorsal thalamus. Only the medial pieces were used in this study, because the lateral pieces do not show consistent axon outgrowth (J. E. Braisted and D. D. M. O'Leary, unpublished data), and the netrin-1 receptors $D C C$ and neogenin are more highly expressed in medial compared to lateral dorsal thalamus (Fig. 2). To isolate floor plate, spinal cords were dissected from rat embryos (E17-E18), and a cut was made along the dorsal midline. The spinal cords were flattened, tissues adjacent to the floor plate were removed, and the floor plate was cut into small pieces.

Aggregates of netrin-1-expressing cells. A netrin-1-secreting 293-EBNA cell line was used (Shirasaki et al., 1996). The parental 293-EBNA cell line transfected with the parental plasmid was used as a control. The cells were harvested, centrifuged to a pellet, and resuspended in 100-300 $\mu \mathrm{l}$ of growth medium (see below). Low melting point agar (2\%) in L15-glucose was added to a $35 \mathrm{~mm}$ dish and allowed to gel. A $1 \mathrm{~cm}$ square was removed, then $35 \mu \mathrm{l}$ of cells and $35 \mu \mathrm{l}$ of molten $2 \%$ agar was added to this cavity and mixed. Small cubes containing cells were then cut from the agar and placed in growth medium and cultured for another 3-5 d, during which the cells continued to proliferate. The cell-filled cubes were cut into smaller pieces before plating.

Collagen gel cocultures. Collagen was prepared from either adult rat tails or $8-10 \mathrm{~cm}$ tails from juvenile rats. Cocultures were set up as follows: 900 $\mu \mathrm{l}$ of collagen solution was mixed with $100 \mu \mathrm{l}$ of $10 \times \mathrm{MEM}$, and 11-18 $\mu \mathrm{l}$ of a $7.5 \%$ solution of sodium bicarbonate. Twenty-five microliters of collagen was pipetted onto the bottom of four well dishes (Nunc, Roskilde, Denmark) and allowed to gel. Explants were then placed onto this base, and $75 \mu \mathrm{l}$ of collagen was added on top. Dorsal thalamic explants and test explants (floor plate or ICZ), or 293 cell aggregates, were positioned $\sim 150-300 \mu \mathrm{m}$ apart. Dorsal thalamic explants were placed adjacent to the medial aspect of the ICZ explants. The orientation of the explant relative to the test explant or 293 cell aggregates was random. Growth medium (GM; $500 \mu \mathrm{l}$ of DMEM/F-12 containing $0.1 \%$ penicillin-streptomycin, $0.6 \%$ D-glucose, $2 \mathrm{~mm}$ glutamine, and $5 \%$ rat serum) was then added. Explants were cultured in a humidified, $37^{\circ} \mathrm{C}, \mathrm{CO}^{2}$ incubator.

Analysis of axon outgrowth in cocultures. After $1.5 \mathrm{~d}$ in vitro, dorsal thalamic axon outgrowth was scored and assigned to one of three categories; explants that had more axon outgrowth either (1)toward or (2) away from the test explant or 293 cell aggregates, or (3) explants that had similar amounts of axon outgrowth on both of these sides and were therefore scored as "symmetric". For dorsal thalamus cultured alone, the near side of the dish was arbitrarily designated "toward" and the far of the dish "away." Cultures were scored blind, and data was analyzed statistically using the $\chi^{2}$ test.

Analysis of axon outgrowth in response to soluble recombinant netrin-1. Dorsal thalamic explants were placed in collagen gels (see above). At the time of plating, purified, recombinant netrin-1 protein produced in 293 cells (Serafini et al., 1994) was directly diluted into the growth medium. After 36-48 hr, cultures were photographed using phase-contrast optics then fixed. The number of axon fascicles leaving dorsal thalamic explants were counted at 100 and $285 \mu \mathrm{m}$ from the explants. At each of these distances, axon fascicles were counted in two $40 \mu \mathrm{m}$ zones showing low axon outgrowth, two zones showing high axon outgrowth, and two zones showing intermediate axon outgrowth. These six numbers were averaged, normalized to $100 \mu \mathrm{m}$, and analyzed with the Student's $t$ test.

Function blocking experiments. At the time of plating, affinity-purified rabbit antibodies raised against domains VI and V of chick netrin-1 protein $(10 \mu \mathrm{g} / \mathrm{ml})$, or control affinity purified nonimmune rabbit IgG (10 $\mu \mathrm{g} / \mathrm{ml})$, were added to dorsal thalamic-floor plate and dorsal thalamic-ICZ cocultures. Cocultures were analyzed as described above.

DiI labeling of the TCA projection. Netrin-1 -/ - and wild-type embryos (E17.5 and E18.5) were perfused with $4 \%$ paraformaldehyde. Brains were removed and dissected in half along the sagittal midline. Crystals of the axonal tracer $1,1^{\prime}$-dioctadecyl 3,3,3',3'-tetramethylindocarbocyanine perchlorate (DiI) (Molecular Probes, Eugene, OR) (Honig and Hume, $1989 a, b)$ were placed in the ventral posterior nucleus of the dorsal thalamus (VP) of one hemisphere to label anterogradely VP axons that project to the neocortex. In the opposite hemisphere of the same animal, crystals of DiI were placed in the region of presumptive primary somatosensory neocortex (S1) to label retrogradely VP neurons that project to it. Crystals were measured on a microscope using a micrometer, and similarly shaped and sized crystals (measuring $\sim 100-200 \mu \mathrm{m}$ in diameter) were placed in S1 of paired littermates (i.e., a mutant homozygous aired with a heterozygous or wild-type littermate-observer blind to genotype).

Brains were left in $4 \%$ paraformaldehyde at $4^{\circ} \mathrm{C}$ or $30^{\circ} \mathrm{C}$ for $\geq 3$ weeks to allow the DiI to diffuse the full length of the axons. Brains were then embedded in $3 \%$ low melting point agar, and 75 or $100 \mu \mathrm{m}$ coronal sections were cut using a vibratome. Sections were counterstained with a $0.02 \%$ solution of the nuclear stain bisbenzimide (Sigma). Labeled cells and axons were photographed under rhodamine (DiI) and UV (bisbenzimide) illumination.

Cortical injection volumes were measured, and pairs of littermates (one wild-type or heterozygote paired with one homozygous mutant) with similarly sized injections (within $10 \%$ of each other; as described in Catalano et al., 1998) in identical regions of S1 were quantitatively compared [E17.5, $n=3$ matched pairs (six animals total); E18.5, $n=2$ matched pairs (four animals total)]. Note that during these quantitative comparisons, it was impossible for the observer to be blind to the genotype of the animal because the altered callosal projection of the netrin-1 mutants is clearly visible in tissue sections used for the comparisons. Serial sections extending throughout the VP nucleus were examined using a ocular graticule on a Nikon FXA microscope and a $10 \times$ objective, and the total number of retrogradely labeled VP neurons in each animal was counted. The number of labeled neurons in wild-type mice was normalized as $100 \%$, and the mutant projection was expressed as a fraction of the wild-type number. The mediolateral width of dorsal thalamus was measured in coronal sections along a line extending perpendicularly from the third ventricle at the midline to the lateral edge of dorsal thalamus using a $4 \times$ lens with an attached calibrated graticule (as described in Catalano and Shatz, 1998).

Distribution of TCAs in the internal capsule. Anterograde DiI labeling and L1 immunostaining were used to examine the distribution of TCAs within the IC. Heads of netrin-1 -/- and wild-type embryos (E15 and P0) were immersion-fixed in $4 \%$ paraformaldehyde. Brains were bisected along 


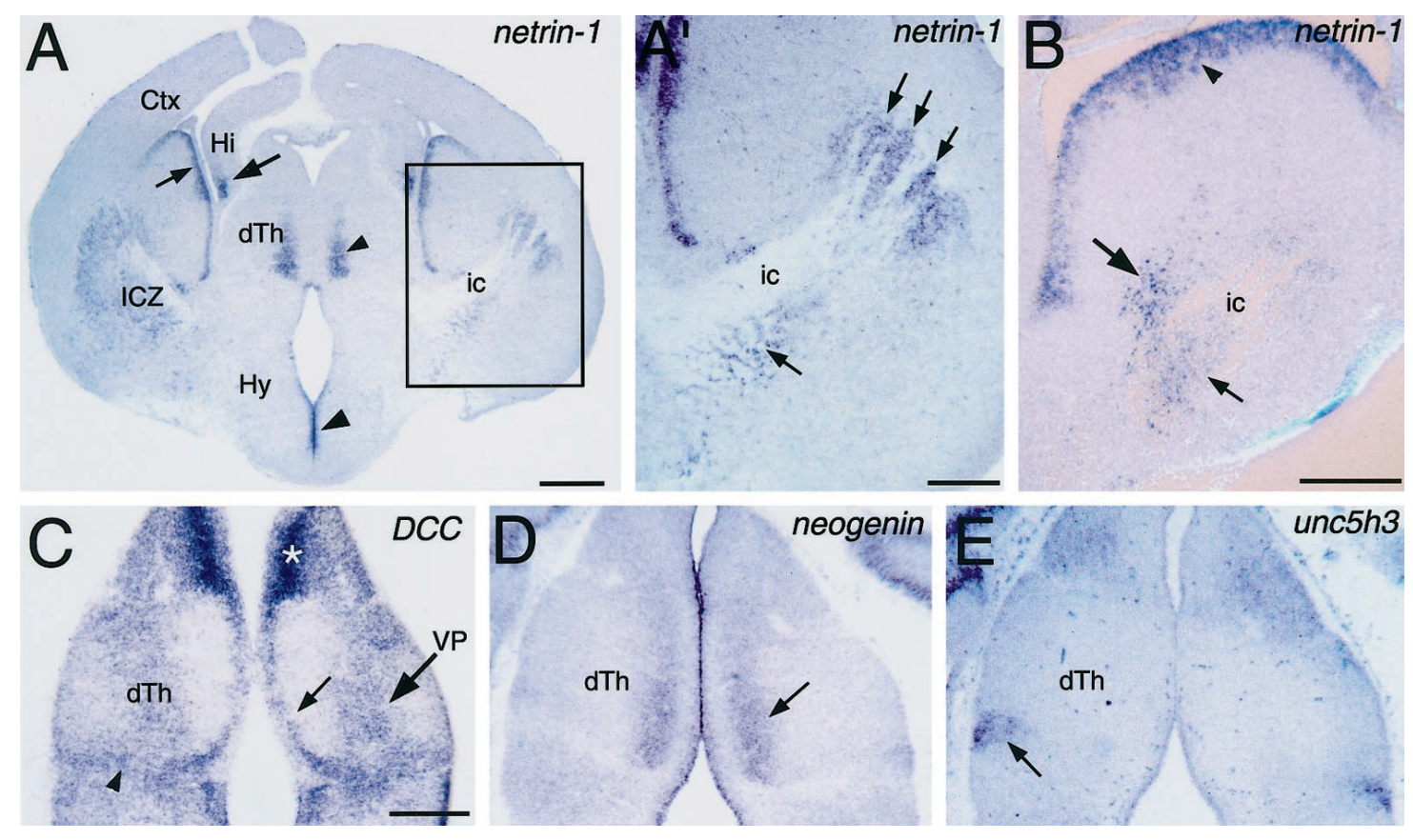

Figure 2. Expression of netrin-1 and netrin receptors in relation to the TCA projection. Coronal sections of E14.5 $\left(A, A^{\prime}\right)$ and E13.5 $(B-E)$ mouse brains showing netrin-1 $\left(A, A^{\prime}, B\right), D C C(C)$, neogenin $(D)$, and unc5h3 $(E)$ expression detected with digoxigenin-labeled riboprobes. Dorsal is up. Lateral is to the right in $A^{\prime}$ and $B$. A, At E14.5, netrin-1 expression is detected in the differentiating mantle zone of the ICZ, as well as in the overlying ventricular zone (small arrow). Netrin-1 is also expressed in the medial part of dorsal thalamus (dTh; small arrowhead), hippocampus (Hi; large arrow), and the hypothalamic $(H y)$ ventricular zone (large arrowhead). $A^{\prime}$, Higher magnification of boxed area in $A$. This is the region used in coculture experiments in Figure 7 (see Materials and Methods). Netrin-1-expressing cells are present in the ICZ (arrows), intermingled with fascicles of TCAs in the internal capsule (ic). B, At E13.5, netrin-1 is expressed in the ICZ, in cells within (small arrow) and surrounding (large arrow) the internal capsule (ic). Expression is also detected in the overlying ventricular zone of the ganglionic eminence (arrowhead). C, DCC expression is detected in the ventricular zone (small arrow) and differentiating mantle zone (large arrow) of dorsal thalamus, and in cells at the dorsal thalamic-ventral thalamic border (arrowhead). The expression within the mantle zone corresponds to the location of the ventral posterior thalamic nucleus $(V B)$. Asterisk indicates high expression in medial habenula. $D$, Neogenin is expressed in medial dorsal thalamus (arrow). E, Unc5h3 is expressed in the lateral dorsal part of ventral thalamus (arrow), but not in dorsal thalamus. Scale bars: $A, 500 \mu \mathrm{m} ; A^{\prime}, B, C, 250 \mu \mathrm{m}$ (bar in $C$ also applies to $D, E$ ).

the midline, and tissues caudal to the diencephalon were removed to expose the caudal surface of dorsal thalamus. Several large DiI crystals were placed into the dorsal thalamus from its caudal surface, either centered on the ventral lateral nucleus medially, or the VP nucleus laterally. Brains were then stored and processed as above. To measure the width of the IC, coronal vibratome sections of P0 netrin-1 -/- and wild-type littermates were examined, and embryos with similarly sized and placed DiI injections were paired ( $n=4$ pairs; eight mice total). The width of the IC containing DiI-labeled axon fascicles was measured perpendicular to the IC, at a point approximately midway along its mediolateral axis.

L1 immunocytochemistry was performed as previously described (Tuttle et al., 1999). Briefly, sections were incubated overnight in rabbit polyclonal anti-L1 antibody (1:2000; a gift from C. Lagenauer), then in biotinylated anti-rabbit IgG (1:200; Vector Laboratories, Burlingame, CA). Sections were treated with $1 \%$ hydrogen peroxide, incubated in avidin-biotin-horseradish peroxidase complex (ABC kit; Vector Laboratories), reacted in $0.3 \% \mathrm{DAB}, 0.03 \%$ hydrogen peroxide in TBS, dehydrated, cleared in xylene, and coverslipped in DPX mountant.

\section{RESULTS}

\section{Expression of netrin-1 relative to the TCA pathway}

Previous studies have reported that netrin-1 is expressed in the striatum of developing rodents (Serafini et al., 1996; Metin et al., 1997). To determine whether netrin-1 may be involved in the pathfinding of TCAs, we examined the expression pattern of netrin-1 relative to the TCA pathway (schematized in Fig. 1). In situ hybridizations were performed on sections of E13.5-E14.5 mouse brains, ages when TCAs are extending into and through the ICZ (Braisted et al., 1999). The pattern of netrin-1 expression at these two ages, and its relationship to the TCA pathway, is very similar.

As TCAs turn from the diencephalon into the ICZ, they immediately encounter a cluster of netrin-1-expressing cells (Fig. $2 A, A^{\prime}$ ). As TCAs course dorsolaterally and rostrally through the ICZ, their pathway remains in close association with netrin-1-expressing cells (Fig. 2A, $A^{\prime}$ ). Particularly striking is the presence of netrin-1expressing cells within and in close proximity to fascicles of TCAs passing through the ICZ (Fig. $2 A^{\prime}, B$ ). Robust netrin-1 expression is also seen in the lateral and medial ganglionic emminences (Fig. 2B). In addition, netrin-1 is expressed in restricted parts of the diencephalon. Netrin-1 is expressed in medial dorsal thalamus, just lateral to the ventricular zone, and in the hypothalamic ventricular zone (Fig. 2A). At caudal levels, netrin-1 is expressed in the mantle zone of ventral hypothalamus (data not shown). Netrin-1 expression is not detected in the neocortex (Fig. 2A), the target of TCAs. These findings show that netrin- 1 is expressed in close association to the path of TCAs as they extend through the ICZ toward the neocortex.

\section{Expression of netrin-1 receptors in dorsal thalamus}

The expression pattern of netrin-1 suggests that it may act as an attractant for TCAs. Therefore, we would expect that DCC and neogenin, receptors that mediate the attractant effects of netrin-1 (Keino-Masu et al., 1996) would be expressed in dorsal thalamus, whereas the unc5 homologs unc5h2 and unc 5 h3, which are thought to mediate the repellent action of netrin-1 (Leonardo and Forst, 1996), might not be. In situ hybridizations were performed on sections through E13.5 dorsal thalamus.

In dorsal thalamus, $D C C$ is expressed in the ventricular zone and in a broad dorsolateral to ventromedial stripe in the mantle zone (Fig. 2C). This stripe includes the VP nucleus, which projects to somatosensory cortex. Expression is also found along the dorsal thalamic-ventral thalamic border, in the region of the zona limitans interthalamica (Fig. 2C). High levels of DCC expression are found elsewhere in the diencephalon, including the habenula and pretectum (Fig. $2 C$ ), and in medial ventral thalamus and hypothalamus (data not shown). Neogenin is expressed in a dorsoventral stripe in medial dorsal thalamus, just lateral to the ventricular zone (Fig. 2D), a pattern similar to that of netrin-1 in dorsal thalamus 

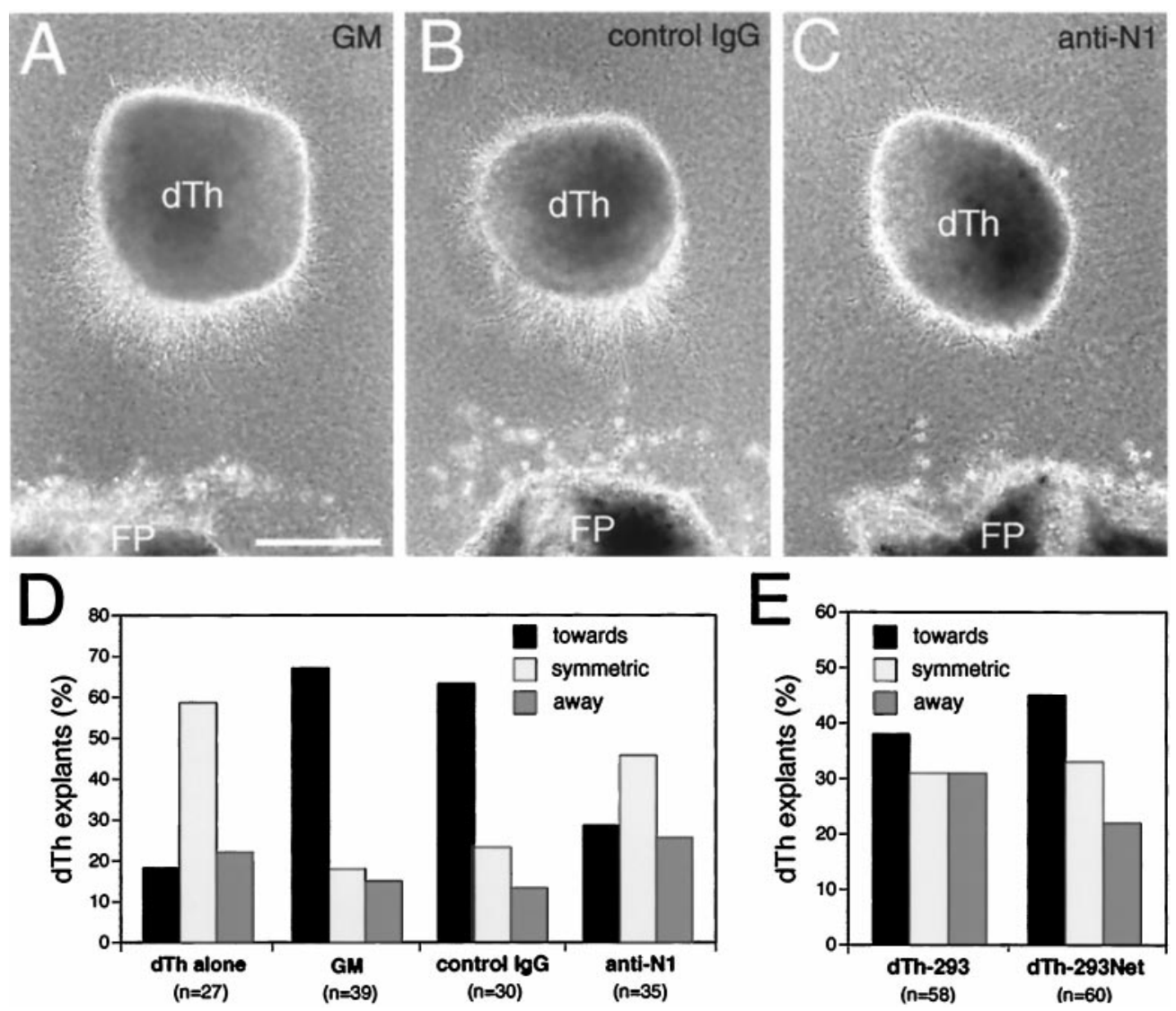

Figure 3. Netrin-1-dependent attraction of dorsal thalamic axons. Explants from the medial half of dorsal thalamus $(d T h$; see Materials and Methods; E13.5-E14.5 mouse) were cocultured for $1.5-2 \mathrm{~d}$ in collagen gels at a distance from floor plate (FP; E17-E18 rat) or aggregates of 293 cells transfected with netrin-1 (dTh$293 \mathrm{Net}$ ) or the parental plasmid as a control (dTh-293). A, Dorsal thalamic axon outgrowth is biased toward FP. $B$, Axon outgrowth from dorsal thalamus is also biased toward FP when nonimmune sera $(10 \mu \mathrm{g} / \mathrm{ml})$ is added to the growth medium $(G M)$. C, Axon outgrowth from dorsal thalamus is no longer biased toward FP when netrin-1 blocking antibodies $(10 \mu \mathrm{g} / \mathrm{ml})$ are added to the GM. $D, E$, Quantification of dorsal thalamic axon responses. $N$ (number of cocultures) values are in parentheses. $D$, When dorsal thalamus is cultured alone (dTh alone), the distribution of axon outgrowth is significantly different from dorsal thalamus cocultured with FP (GM; $p<0.0002, \chi^{2}$ test). When dorsal thalamus is cocultured with FP in the presence of netrin-1 blocking antibodies (anti-N1), the distribution of axon outgrowth is significantly different from dorsal thalamus cultured in GM or GM with added nonimmune sera (control IgG; $p<0.004$ and 0.02 , respectively, $\chi^{2}$ test). Dorsal thalamic axon outgrowth in cocultures with control IgG was not significantly different from that with GM alone $\left(p=0.85, \chi^{2}\right.$ test $)$. $E$, Although there is a trend for dorsal thalamic axon outgrowth to be biased toward netrin-1expressing cells (dTh-293Net) compared to control 293 cells $(d T h-293)$, these differences are not significant $\left(p=0.5, \chi^{2}\right.$ test). Scale bar, $250 \mu \mathrm{m}$.
(Fig. 2A). Neogenin expression is also present in the ventral thalamic ventricular zone (data not shown).

In contrast to $D C C$ and neogenin, neither unc5h2 (data not shown) nor unc5h3 (Fig. 2E) are expressed in dorsal thalamus, although both are expressed elsewhere in the diencephalon. Unc5h2 is expressed in anterior parts of ventral thalamus, the ventral thalamic ventricular zone, and the pretectum (data not shown). Unc $5 h 3$ expression is present in a region of ventral thalamus that will develop into the ventral lateral geniculate nucleus (Fig. 2E) and in scattered cells in the hypothalamus (data not shown).

These data demonstrate that at the time TCAs are extending through the ICZ, the attractant netrin-1 receptors $D C C$ and neogenin are expressed in dorsal thalamus, but the repellent netrin-1 receptors $u n c 5 h 2$ and $u n c 5 h 3$ are not. Taken together with the expression pattern of netrin-1, these findings suggest that netrin-1 may act to attract TCAs into and/or through the ICZ.

\section{Netrin-1 attracts dorsal thalamic axons in vitro}

To test the action of netrin- 1 on TCAs, we cocultured at a distance in three dimensional collagen gels (Lumsden and Davies, 1983; Tessier-Lavigne et al., 1988) explants of medial dorsal thalamus (which expresses high levels of DCC/neogenin) with explants of floor plate, which expresses netrin-1, or aggregates of 293 cells stably transfected with netrin-1 cDNA (see Materials and Methods). Dorsal thalamic explants were prepared from E13.5-E14.5 mouse embryos, ages when TCAs are growing through the ICZ. Explants of floor plate were prepared from E17-E18 rat spinal cord, ages when the floor plate expresses netrin-1 (Yee et al., 1999).

Dorsal thalamic explants cultured alone typically exhibit symmetric axon outgrowth (Fig. $3 D$ ). In contrast, dorsal thalamic explants cocultured at a distance from floor plate exhibit a statistically significant bias in axon outgrowth toward the floor plate (Fig. 3A,D). To determine whether netrin-1 is responsible for this bias, dorsal thalamic explants were cocultured at a distance from floor plate in the presence of netrin-1 blocking antibodies, or a control IgG. When cocultured in the presence of a control $\mathrm{IgG}$, dorsal thalamic axon outgrowth still shows a statistically significant bias toward the floor plate (Fig. $3 B, D$ ). In contrast, when cocultured in the presence of netrin-1 blocking antibodies, the biased growth of dorsal thalamic axons toward floor plate is abolished (Fig. 3C,D). Thus, the floor plate releases a diffusible activity that attracts dorsal thalamic axons in vitro, and this activity is likely to be netrin-1. Dorsal thalamic explants cocultured with aggregates of netrin-1-transfected 293 cells also show biased outgrowth toward the netrin-1 source (Fig. 3E). This biased outgrowth is less robust than that observed in the floor plate cocultures, but is stronger, albeit not statistically significant, than that exhibited by dorsal thalamic explants cocultured with aggregates of parental 293 cells (Fig. 3E). Nonetheless, when taken together, these coculture experiments suggest that netrin-1 acts in vitro as an attractant for dorsal thalamic axons.

\section{TCAs are disorganized and abnormally restricted in the IC of netrin-1 -/- mice}

The findings described above suggest that netrin-1 may act in vivo to attract TCAs into the ICZ and direct their growth through it toward the cortex. Therefore, we analyzed the development of the TCA projection in netrin-1 -/ - mice using anterograde and retrograde DiI axon tracing and L1 immunohistochemistry. The distribution of labeled axons was compared between netrin-1 $-/-,+/-$, and $+/+$ littermates. Because no significant differences were found between the $+/+$ and $+/-$ embryos, they will both be referred to as wild-type.

To anterogradely label a substantial proportion of TCAs, large crystals of DiI were placed into dorsal thalamus. At E15.5, TCAs have extended through the IC and entered the neocortex in both wild-type (Fig. 4A-C) and netrin-1 -/ (Fig. 4D-F) mice. Labeled axons in the IC at this age consist predominantly of TCAs, because only a small number of retrogradely labeled neurons are found in 

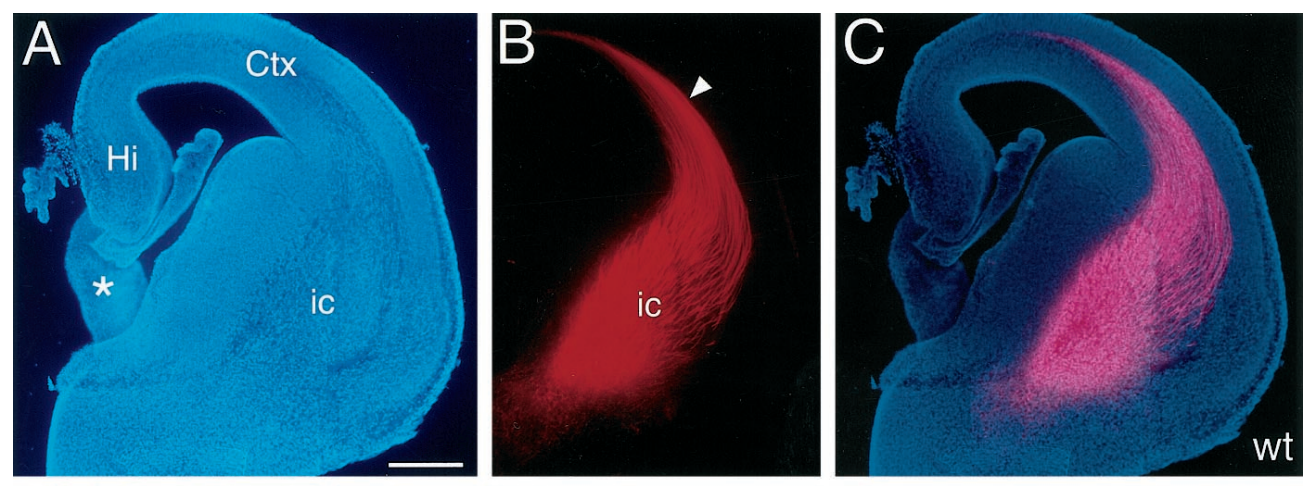

Figure 4. The path of TCAs is abnormally restricted in E15 netrin-1 -/ - mice. Coronal sections of E15 mouse brains from wild-type $(A-C)$ and netrin-1 -/$(D-F)$ littermates with DiI implanted into dorsal thalamus, showing DiI-labeled TCAs $(B, E)$, bisbenzimide counterstain of the same sections $(A, D)$, and computer-generated overlays of the DiI and bisbenzimide images $(C, F)$. In wildtype mice, TCAs are broadly and evenly distributed in the internal capsule (ic). In netrin-1 - /- mice, the TCA pathway is restricted dorsomedially, and fascicles of TCAs appear disorganized; the ventrolateral portion of the pathway is devoid of TCA fascicles $(E, F$, arrows). The magnitude of the TCA projection is also reduced in the netrin-1 - /- mice compared to their wild-type littermates. In both wild-type and netrin-1 -/- littermates, TCAs have entered the intermediate zone/subplate underlying the neocortex (Ctx; B, E, arrowheads). Asterisks in $A$ and $D$ indicate the rostral portion of the thalamus. Hi, Hippocampus; St, striatum. Scale bar, $300 \mu \mathrm{m}$. Dorsal is to the top, and lateral is to the right.
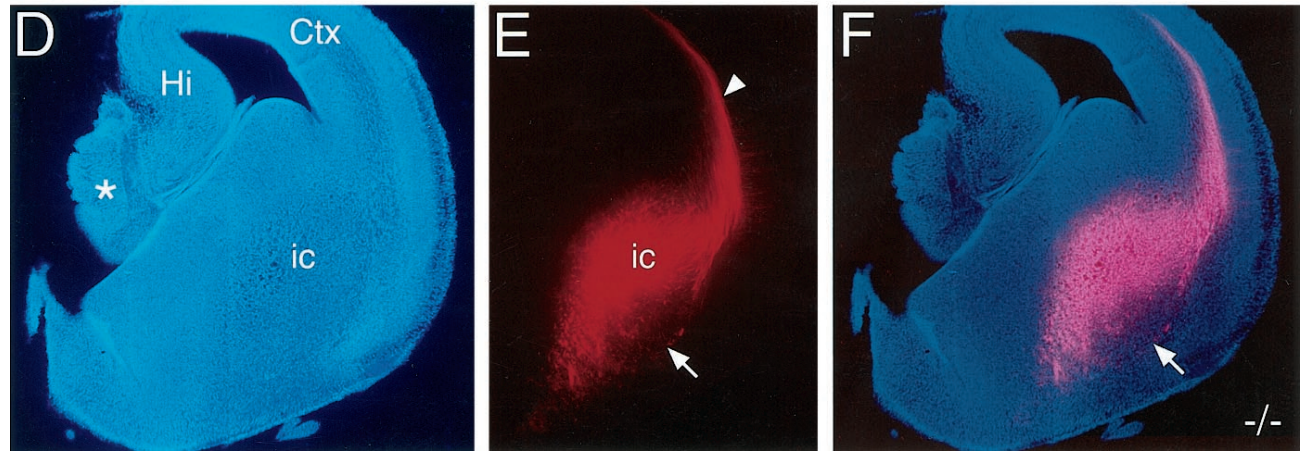

the neocortex (data not shown). The trajectory of TCAs within the proximal portion of their pathway, which includes their ventral extension along the lateral aspect of the ventral thalamus and their lateral turn into the ICZ, is indistinguishable between wild-type and netrin-1 -/- littermates (data not shown). However, more distally along their pathway, where TCAs would normally encounter the domain of netrin-1 expression in the ICZ, they exhibit aberrancies in netrin-1 $-/-$ mice. Fewer labeled fascicles of TCAs are evident in netrin-1 - /- mice compared to wild type, and their distribution is much more restricted; in wild-type mice the labeled fascicles are broadly distributed over the ICZ (Fig. $4 C$ ), but in the netrin-1 $-/-$ mice few if any are found in its ventrolateral part (Fig. $4 F$ ). These observations indicate that netrin- 1 is not required for TCAs to turn and enter the ICZ, but is required for their proper distribution within the ICZ.

To determine whether the restricted distribution of TCAs within the ICZ of netrin-1 $-/-$ mice persists, we examined the TCA projection in netrin- $1-/-$ and wild-type mice at $\mathrm{P} 0$, the age when netrin-1 mutants die (Fig. 5). At this age, DiI placed in dorsal thalamus anterogradely labels TCAs, and in addition retrogradely labels corticothalamic axons. In P0 wild-type mice, TCAs have reached the neocortex and invaded the cortical plate (Fig. 5B,C). The labeled TCA fascicles extend dorsolaterally through the IC and are distributed in an orderly manner throughout most of the striatum. Superimposed on the dorsolaterally projecting axon fascicles is a more-or-less uniform diffuse labeling indicative of axon terminations (Fig. 5B,C). In netrin-1 -/- mice, the diff use terminal labeling is present throughout the striatum as in wild type. However, in contrast to wild-type, in netrin-1 -/- mice labeled TCA fascicles are abnormally restricted to the dorsomedial part of the striatum (Fig. 5E,F). This restricted distribution in netrin-1 -/mice is not attributable to a reduced size of the striatum. Compared to paired wild-type littermates, in netrin-1 -/- mice the width of the striatum is on average only $2.5 \%$ smaller, but the width of the distribution of labeled axon fascicles is reduced by $49 \%$ (Fig. $5 I$ ). In conclusion, the TCA pathway through the striatum is abnormally narrow in netrin-1 $-/-$ mice throughout the period of TCA pathfinding and is restricted to dorsomedial striatum.

To control for potential differences in DiI placements, the IC phenotype was verified by immunostaining sections of netrin-1 -/and wild-type P0 littermates with an L1 antibody, which labels both TCAs and corticothalamic axons in the IC at this age. In sections at the level shown in Figure 5, $E$ and $F$, the appearance and distribution of DiI-labeled and L1-labeled (L1; data not shown) axons is similar. However, in the L1-labeled preparations, we also noted a striking phenotype in sections caudal to that shown in Figure 5, E and $F$. The phenotype at this caudal-most level was not as obvious in the DiI preparations, because the intensity of DiI label obscured the defect (data not shown). In wild-type mice, the L1immunostained axon fascicles in caudal-most IC are relatively fine, oriented more-or-less parallel to one another, and evenly distributed within the IC (Fig. 5G). In contrast, in netrin-1 -/- mice, the L1-immunostained axon fascicles are larger, less numerous, abnormally organized, and restricted in their distribution (Fig. $5 H$ ). In addition, because L1 also labels corticothalamic axons, it is likely that they exhibit abnormalities similar to those of TCAs in the netrin-1 -/- mice.

Together, these data demonstrate that in the absence of netrin-1, TCAs can extend out of dorsal thalamus, through the ventral thalamus and turn as they approach hypothalamus to enter the ICZ in an apparently normal fashion. However, within regions of ICZ that normally would express netrin-1, fascicles of TCAs are disorganized and abnormally restricted to the dorsomedial half of the ICZ/striatum. The path of corticothalamic axons appears to be similarly aberrant. These data suggest that netrin- 1 provides a favorable growth substrate for TCAs within the ICZ, and that in the absence of netrin-1, the ICZ is less permissive for TCA growth.

\section{The TCA projection to neocortex is reduced in netrin-1 mutant mice}

The finding that the distribution and organization of fascicles of TCAs in the IC is abnormal in netrin- $1-1-$ mice suggests that the ICZ is less permissive for TCA growth in the absence of netrin-1. These findings raise the possibility that fewer TCAs reach the neocortex in netrin-1 - /- mice and that their area-specific targeting may be abnormal. We examined these possibilities by using anterograde and retrograde labeling of the TCAs originating from the VP thalamic nucleus, which projects to somatosensory (parietal) cortex.

A specific population of TCAs were anterogradely labeled by injecting DiI into VP in netrin-1 $-/-$ mice and their wild-type littermates. In both genotypes, the labeled VP axons extend through the IC and terminate specifically in parietal cortex (Fig. 6). Thus, even though in netrin-1 $-/-$ mice the TCA projection 

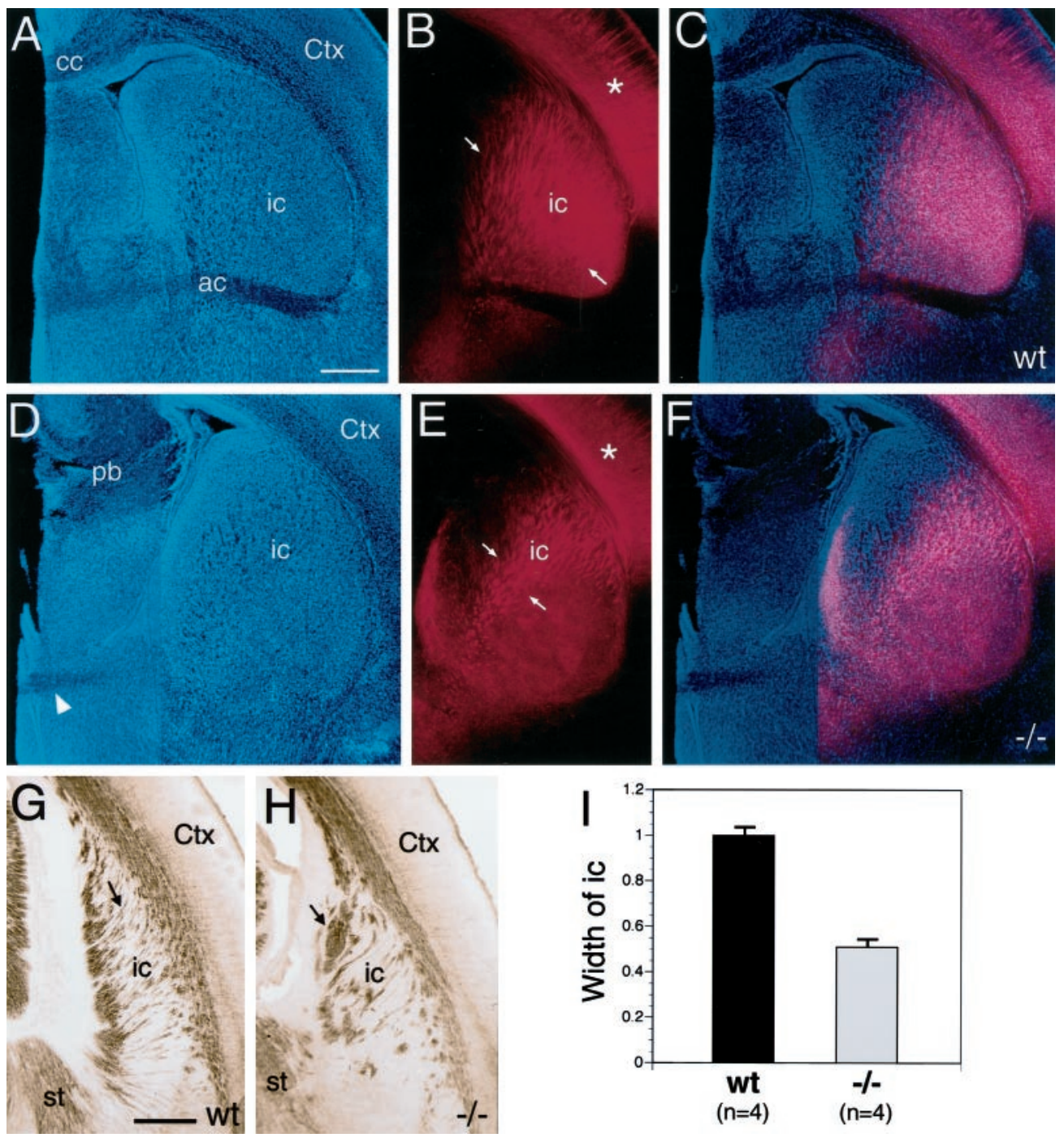

through the ICZ/IC is disorganized and restricted in its distribution, TCAs appear to exhibit normal area-specific targeting. However, the magnitude of the TCA projection to cortex is obviously reduced in the netrin-1 -/- mice compared to their wild-type littermates. The extent of this reduction is variable; in some netrin-1 $-/-$ mice we observe a severe reduction of the projection (Fig. $6 D, D^{\prime}, E, E^{\prime}$ ), whereas in others the projection appears to be only slightly reduced (Fig. $6 B, B^{\prime}$ ). Individual labeled axons of the more severely affected netrin-1 $-/-$ mice also appear abnormal (Fig. $6 F, F^{\prime}, \mathrm{E} 18.5$ ). In these cases, axon branches do not extend as far into the cortical plate as in wild-type littermates, or in normal mice of this age (Catalano et al., 1991, 1996; Cohen-Tannoudji et al., 1994), suggesting that TCA ingrowth into the neocortex may be delayed in the netrin-1 mutants.

To corroborate the anterograde findings and quantify the reduction in the TCA projection in netrin-1 - / - mice, DiI was injected at late embryonic ages into somatosensory cortex in wild-type and netrin-1 -/- littermates to retrogradely label its TCA input (Fig. $6 G, H)$. As in wild-type mice, these injections retrogradely label VP neurons in the netrin-1 -/- mice. However, on average, the number of labeled VP neurons in netrin-1 $-/-$ mice is reduced by $\sim 40 \%$ compared to wild type (Fig. $6 I$ ). As observed with anterograde labeling, the reduction in the TCA projection varies among netrin-1 $-/-$ cases, ranging from a reduction of $30-90 \%$ in the number of labeled VP neurons compared to injection-matched wild-type littermates (see Materials and Methods). It is unlikely that cell death in the dorsal thalamus contributes prominently to this reduction, because the cytoarchitecture and the mediolateral
Figure 5. The internal capsule is abnormally narrow, and fascicles of TCAs are disorganized in P0 netrin-1 - /- mice. Coronal sections of $\mathrm{P} 0$ mouse brains from wild-type $(A-C)$ and netrin-1 $-/-(D-F)$ littermates with DiI implanted into dorsal thalamus $(d T h)$, showing DiI-labeled processes $(B, E)$, bisbenzimide counterstain $(A, D)$, and computer-generated overlay of DiI and bisbenzimide images $(C, F)$. In wild-type mice, the fascicles of TCAs $(B$, $C$, between arrows) are distributed throughout most of the striatum. In P0 netrin-1 - - mice, the fascicles of TCAs ( $E, F$, between arrows) are restricted to dorsomedial striatum. In both wild-type and netrin-1 - - - littermates, TCAs have extended into neocortex (Ctx) and have begun to invade the cortical plate $(B, E$, asterisks). $G, H$, Coronal sections through the caudal part of the internal capsule (ic) of P0 wild-type $(G)$ and netrin-1 - $-(H)$ littermates immunolabeled with an L1 antibody and visualized with a peroxidaseconjugated secondary antibody. In wildtype mice, the L1-immunolabeled axon fascicles are relatively thin and extend roughly parallel to one another $(G, a r-$ row). In netrin-1 - / - mice, the L1immunolabeled axon fascicles are thicker, less numerous, and abnormally organized $(H$, arrow $)$. I, Quantification of the distribution of DiI-labeled fascicles of TCAs within the internal capsule of P0 wild-type and netrin-1 -/- mice. For quantification, mice were paired according to the size and placement of DiI in the dorsal thalamus. The width of the internal capsule (i.e., the distribution of labeled fascicles of TCAs, measured between arrows in $B, E$ ) within the striatum in netrin-1 $-/-$ mice is significantly reduced, being $\sim 50 \%$ of that in paired wild-type littermates $(I ; n=4$ littermate pairs, $p<0.001$ Student's $t$ test). $c c$, Corpus callosum; $p b$, Probst bundle; $w t$, wild type. Scale bars: $A, 400 \mu \mathrm{m}$ (also applies to $B-F$ ); $G, 300 \mu \mathrm{m}$ (also applies to $H$ ). Dorsal is to the top, and lateral is to the right. dimension of dorsal thalamus in netrin-1 -/- and wild-type littermates are not significantly different ( $p=1$ for E18.5, $p=0.37$ for E17.5; data not shown). These data confirm the findings obtained with anterograde axon labeling that the loss of netrin-1 in the ICZ results in a significant, but variable, reduction in the TCA projection.

\section{ICZ releases an attractant activity for dorsal thalamic axons distinct from netrin-1}

Previous studies have shown that the ICZ releases an activity that attracts dorsal thalamic axons at a distance in collagen gels (Braisted et al., 1999). To test whether netrin-1 is the ICZ attractant, we cocultured explants of medial dorsal thalamus and ICZ from E13.5-E14.5 mice in collagen gels and attempted to block the ICZ attractant effect by adding to the culture medium a netrin-1blocking antibody, or as a control, a nonimmune serum. In the presence of nonimmune serum $(10 \mu \mathrm{g} / \mathrm{ml})$, dorsal thalamic axon outgrowth is biased toward the ICZ (Fig. 7A,B). The bias in dorsal thalamic axon outgrowth toward the ICZ is diminished in the presence of the netrin-1 blocking antibody $(10 \mu \mathrm{g} / \mathrm{ml})($ Fig. $7 B)$, but this change is not statistically significant $(p=0.7)$. These data suggest that the ICZ releases an attractant activity for dorsal thalamic axons distinct from, and perhaps in addition to, netrin-1. Although these in vitro data are consistent with our in vivo findings that in netrin-1 mutants, TCAs extend through the ICZ, albeit in reduced numbers, we cannot rule out the possibility that the blocking antibody is not completely effective at neutralizing the action of netrin-1. 

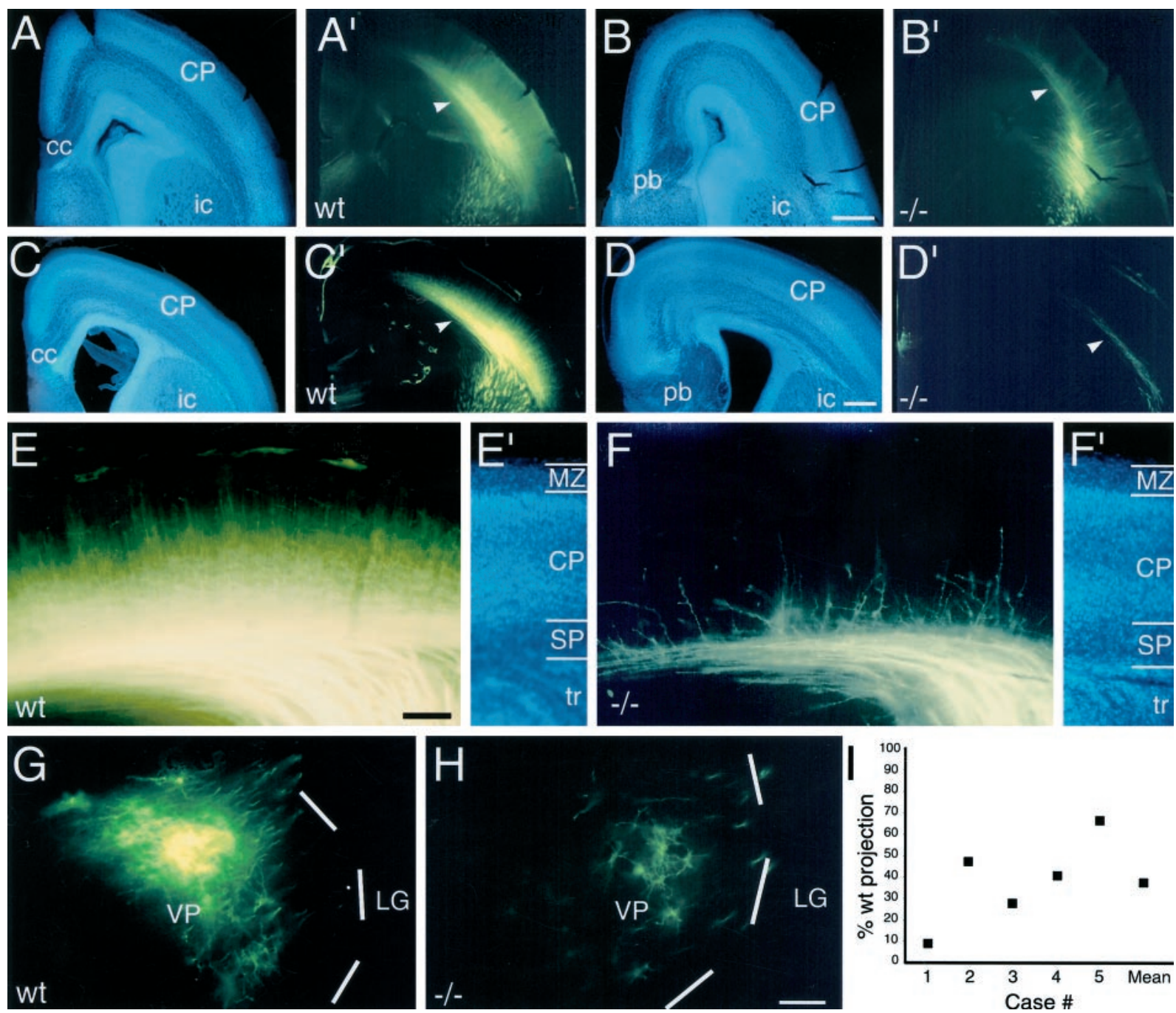

Figure 6. The TCA projection is variably reduced in netrin-1 - / mice. Coronal sections of E17.5 $\left(A, B^{\prime}\right)$ and E18.5 $(C-H)$ mouse brains from wild-type $\left(A, A^{\prime}, C, C^{\prime}, E, E^{\prime}, G\right)$ and netrin-1 - $-\left(B, B^{\prime}, D, D^{\prime}, F, F^{\prime}, H\right)$ littermates, showing DiI-labeled cell bodies $(G, H)$, axons $\left(A^{\prime}-D^{\prime}, E, F\right)$, and bisbenzimide counterstain of the same sections $\left(A-D, E^{\prime}, F^{\prime}\right)$. DiI was implanted into the ventroposterior thalamic nucleus $(V P)$ to anterogradely label VP axons $\left(A^{\prime}-D^{\prime}\right.$, $E, F)$, or into somatosensory cortex to retrogradely label VP neurons $(G, H)$. I, Quantification of retrogradely labeled VP neurons. In wild-type animals at E17.5 $\left(A^{\prime}\right)$ and E18.5 $\left(C^{\prime}\right)$, TCAs pass through the internal capsule (ic) to reach cortex, extend intracortically within the subplate (SP; $A^{\prime}, B^{\prime}$, arrowheads), and extend branches into the cortical plate $(C P)$. In contrast, in netrin-1 - / - littermates at E17.5 $\left(B^{\prime}\right)$ and E18.5 $\left(D^{\prime}\right)$, the TCA projection is reduced $\left(B^{\prime}\right.$, $D^{\prime}$, arrowheads). E, $F$, Higher magnification images of the TCA projections shown in $C^{\prime}$ and $D^{\prime}$, respectively. In the wild-type mouse, a substantial population of anterogradely labeled VP axons is present in the subplate, and their branches densely invade the cortical plate (E). In the netrin-1 -/littermate, fewer labeled VP axons reach the subplate, and fewer branches extend into the cortical plate $(F)$. $G, H$, The number of VP neurons retrogradely labeled by DiI injected into somatosensory cortex in netrin-1 - / - mice $(H)$ is considerably reduced compared to their matched wild-type littermates $(G)$. Dashed white lines mark the lateral border of the VP nucleus. $I$. The number of retrogradely labeled VP neurons in netrin-1 $-/-$ mice plotted as a percentage of the number in wild-type littermates paired for injection size and location. Case \# 1 corresponds to the pair shown in $G$ and $H$. The mean value for the five cases is also plotted. $L G$, Lateral geniculate nucleus; $M Z$, marginal zone; $t r$, thalamic radiations. Scale bars: $A-D^{\prime}, 200 \mu \mathrm{m}$ (also applies to $A-C$ and $A^{\prime}-C^{\prime}$ ); $E, F^{\prime}, 100 \mu \mathrm{m} ; G, H, 100 \mu \mathrm{m}$. Dorsal is to the top, and lateral is to the right.

\section{Netrin-1 enhances dorsal thalamic axon growth in vitro}

The restricted distribution of TCAs in the ICZ/striatum and the reduced number of TCAs that reach the cortex suggest that netrin-1 enhances or facilitates TCA growth through this distal portion of their subcortical pathway. Therefore, we tested in vitro whether netrin-1 enhances dorsal thalamic axon outgrowth. Explants of E13.5-E14.5 medial dorsal thalamus were cultured alone in collagen gels with or without soluble recombinant netrin-1 protein added to the growth medium. Dorsal thalamic axon outgrowth observed under control conditions (Fig. $8 A$ ) is substantially enhanced in the presence of netrin-1 (Fig. $8 B$ ). The addition of netrin-1 results in a statistically significant increase in both the number (Fig. $8 C ; p<0.0001$ ) and the length (Fig. $8 D ; p<0.01$ ) of dorsal thalamic axon fascicles compared to control conditions. Thus netrin-1 has a significant growth-promoting effect on dorsal thalamic axons in vitro.

\section{DISCUSSION}

We have used gene expression analyses, in vitro axon guidance assays, and an analysis of the development of the TCA projection in netrin-1 -/ - mice, to assess the hypothesis that netrin-1 is a positive regulator of TCA pathfinding through the ICZ, a domain in the ventral telencephalon that TCAs traverse en route to the cortex. We show that netrin-1 is expressed at the appropriate time and place by cells in the ICZ to influence TCA axon pathfinding through the ventral telencephalon and that DCC and neogenin, netrin receptors implicated in mediating axonal attraction to netrin (Keino-Masu et al., 1996), are expressed at the appropriate time in dorsal thalamus, the origin of TCAs. Our in vitro experiments indicate that netrin-1 can attract as well as enhance the outgrowth of TCAs, but that it is not solely responsible for the attractant effect of the ICZ on TCAs reported by Braisted et al. (1999). In netrin-1 $-/-$ mice, we find that TCAs project more-or-less along their 


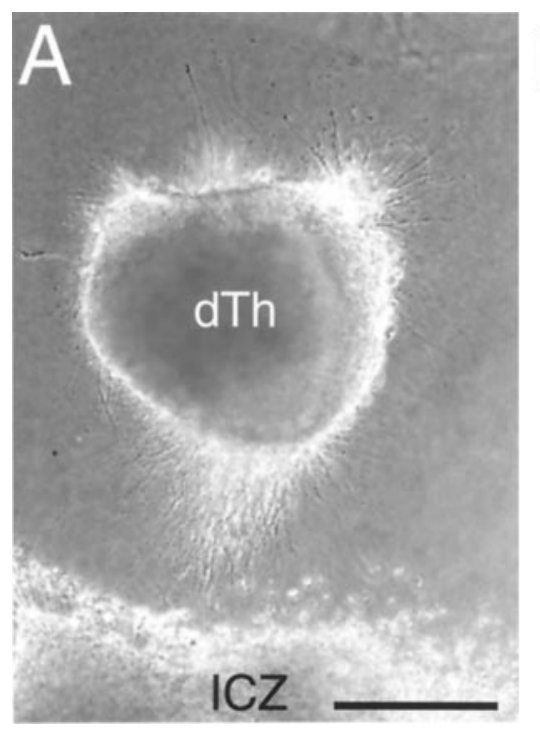

B

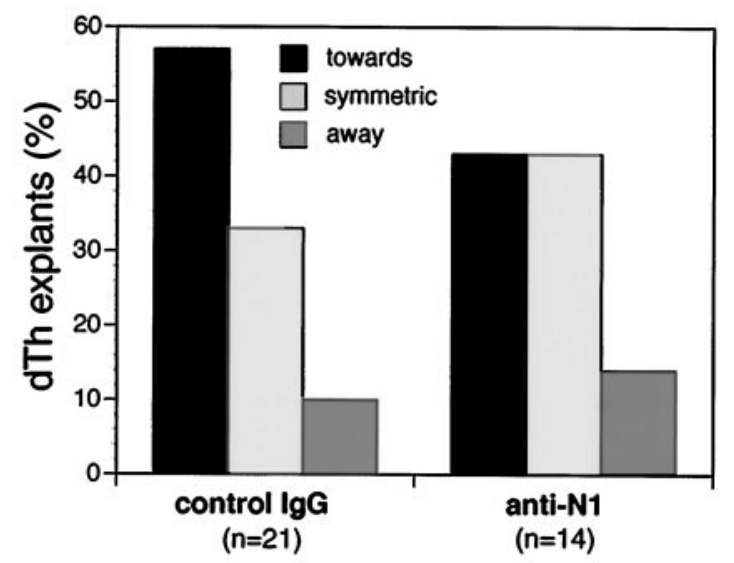

Figure 7. The ICZ releases an attractant activity for dorsal thalamic axons that is distinct from netrin-1. Explants from the medial half of dorsal thalamus $(d T h$; E13.5E14.5 mouse) were cocultured for $1.5-2 \mathrm{~d}$ in collagen gels at a distance from the location of the ICZ in explants of ventral telencephalon (E13.5-E14.5 mouse). Netrin-1 blocking antibodies (anti-N1; $10 \mu \mathrm{g} / \mathrm{ml}$ ) or nonimmune sera (control IgG; $10 \mu \mathrm{g} / \mathrm{ml}$ ) were added to the growth medium at the time of plating. The number of cocultures $(n)$ is indicated in parentheses. When cultured in the presence of control $\mathrm{IgG}$, dorsal thalamic axon outgrowth is biased toward the ICZ $(A, B)$. Addition of netrin-1 blocking antibodies diminishes the percentage of dorsal thalamic explants showing biased outgrowth toward the ICZ (B, anti-N1); however, the distributions of dorsal thalamic axon outgrowth in the control IgG and anti-N1 cocultures are not significantly different ( $p=0.7, \chi^{2}$ test). Scale bar, $250 \mu \mathrm{m}$.
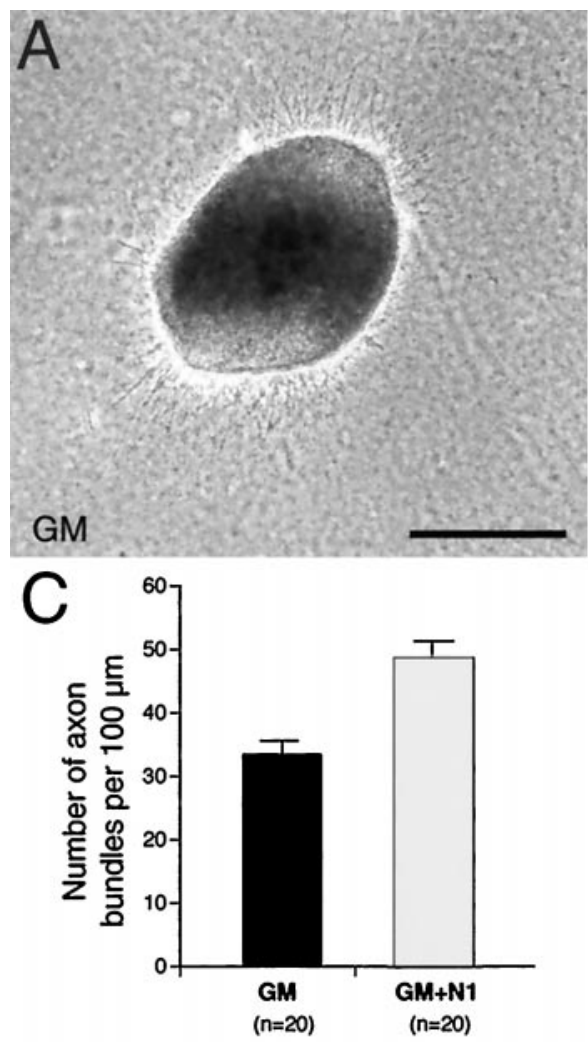

$100 \mu \mathrm{m}$ from explant

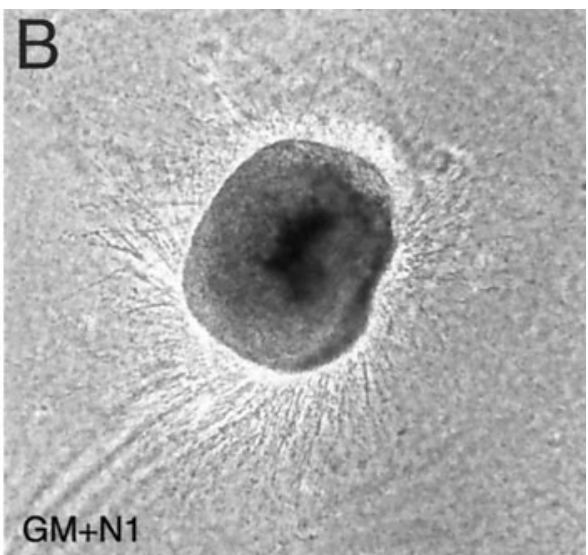

D

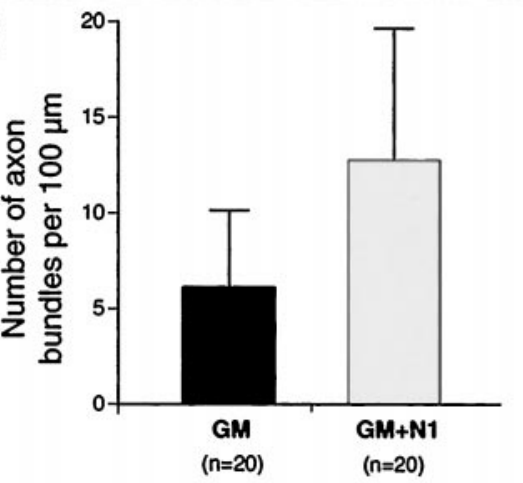

$285 \mu \mathrm{m}$ from explant
Figure 8. Netrin-1 enhances dorsal thalamic axon outgrowth. Explants from the medial half of dorsal thalamus $(d T h)$ were cultured in collagen gels for $1.5-2 \mathrm{~d}$ either in growth medium $(A, G M)$ or in growth medium supplemented with soluble recombinant netrin-1 $(B, G M+N 1 ; 400 \mathrm{ng} / \mathrm{ml}) . C, D$, Quantification of axon outgrowth at $100 \mu \mathrm{m}(C)$ and $285 \mu \mathrm{m}(D)$ from dorsal thalamic explants. The number of cultures analyzed is given in parentheses. A greater number of axons are present at both 100 $\mu \mathrm{m}(C)$ and $285 \mu \mathrm{m}(D)$ in the cultures to which netrin-1 had been added. The differences in axon outgrowth between the two culture types are significantly different $(p<0.0001$ at $100 \mu \mathrm{m} ; p<0.01$ at $285 \mu \mathrm{m}$, Student's $t$ test). Scale bar, $250 \mu \mathrm{m}$. normal path and are able to reach the cortex. Therefore, netrin-1 is not required to reorient TCAs from their ventrally directed path in the diencephalon, laterally into the ICZ. However, the number of TCAs that reach the cortex is reduced in netrin $-1-/-$ mice, and their pathway through the ICZ is disorganized and abnormally narrow. Taken together, our findings show that netrin- 1 is required for the proper development of the TCA projection and indicate that it acts combinatorially with other guidance cues to control TCA pathfinding.

Our in vitro coculture experiments demonstrate that dorsal thalamic axons are attracted at a distance toward floor plate explants. It is likely that netrin-1 is responsible for this attraction because (1) it expressed and released by floor plate (Serafini et al., 1996), (2) the attractant effect of floor plate on dorsal thalamic axons is abolished by the addition of netrin-1-blocking antibodies, and (3) dorsal thalamus expresses $D C C$ and neogenin, two receptors that mediate attractant effects of netrin-1 (Keino-Masu et al., 1996). In addition, dorsal thalamic cells are responsive to netrin- 1 because overall axon outgrowth from dorsal thalamic explants is significantly enhanced when soluble netrin- 1 is added to the culture medium. Therefore, we expected that dorsal thalamic axons would also be attracted by netrin-1-expressing 293 cells. However, we found that although there is a trend for these cells to attract dorsal thalamic axons, it is not statistically significant. Although this raises the possibility that netrin-1 may not by itself have an attractant effect on TCAs, we think that a more likely explanation is that the 293 cells may not produce sufficient netrin-1 to show a clear attractant effect above the normally robust background outgrowth of axons from dorsal thalamic explants in our cultures. In addition, floor plate cells may secrete an additional factor or factors that 
potentiate the action of netrin-1, such as the "netrin-synergizing activity" or NSA (Serafini et al., 1994), which may not be produced by 293 cells. Whatever the explanation, our other in vitro findings show that netrin-1 can stimulate dorsal thalamic axon outgrowth and may provide a directional cue for them, at least in vitro, as suggested by the blocking effect of netrin-1 antibodies on the biased growth of dorsal thalamic axons toward floor plate.

Our analyses of netrin-1 $-/-$ mice show that fascicles of TCAs are disorganized in the IC, their pathway through the IC is abnormally narrow, and fewer TCAs reach the neocortex. Several findings suggest that these mutant phenotypes are likely caused by the direct effect of the loss of netrin-1 in the ICZ on TCA growth. First, at the time TCAs grow through the ICZ, netrin-1 is expressed in it in close association to TCAs, and DCC and neogenin are expressed in dorsal thalamus. In addition, our in vitro findings indicate a direct effect of netrin-1 on TCAs. However, we cannot rule out that the in vivo defects in the TCA projection in netrin-1 $-/-$ mice may be attributable in part to a defect in corticothalamic axon pathfinding. The "handshake hypothesis" put forward by Molnar and Blakemore postulates that TCAs use cortical efferent axons as a scaffold to navigate from the IC to the neocortex, and vice versa (Blakemore and Molnar, 1990; Molnar and Blakemore, 1995; Molnar et al., 1998). Other studies have shown that embryonic cortical axons orient both in vivo (Richards et al., 1997) and in vitro (Metin and Godement, 1996; Richards et al., 1997) toward a region of the embryonic ventral telencephalon that contains the rostral-most portion of the ICZ, and in vitro assays demonstrate that the implied ICZ chemoattractant activity can be mimicked by netrin-1 (Metin and Godement, 1996; Richards et al., 1997). Thus, because cortical axons respond to netrin-1, it may contribute to the extension of cortical axons through the IC; therefore some of the defects that we observe in the TCA projection in netrin-1 -/- mice may be secondary to defects in the cortical efferent projection. However, we should note that a substantial cortical efferent projection does extend through the IC in netrin-1 -/- mice (Braisted and O'Leary, unpublished observations).

An interesting related issue is that even though we find that fascicles of TCAs, and likely corticothalamic axons, are disorganized within the IC, TCAs nonetheless exhibit area-specific targeting. Although our analysis of this issue is limited, these findings suggest that the aberrant deployment of TCAs within the IC, and likely a similar aberrancy in cortical efferent axons (which have been hypothesized to guide TCAs to their correct cortical targets) (Blakemore and Molnar, 1990; Molnar and Blakemore, 1995; Molnar et al., 1998), does not significantly compromise the subsequent pathfinding and area-specific targeting of TCAs within the cortex.

Our findings suggest that a major role of netrin- 1 in the ICZ is to promote the growth of TCAs through the ventral telencephalon. This interpretation is suggested by both our in vitro finding that soluble netrin-1 applied to the medium enhances axon outgrowth from dorsal thalamic explants, and by our in vivo analyses that reveal a reduction in the TCA projection in netrin-1 $-/-$ mice. This reduced projection is unlikely attributable to the loss of TCA projection neurons, because we detect no differences in the cytoarchitecture or size of dorsal thalamus between netrin-1 $-/-$ and wild-type mice, even in those mutants with the most extreme reduction in the TCA projection $(90 \%$ reduced compared to wild type).

Anterograde labeling in netrin-1 -/- mice shows that as a population TCAs make their normal turn from the diencephalon to enter the ICZ. This observation suggests that the primary cause of the reduced projection is the aberrant extension of TCAs through the ICZ, which would also be most consistent with our in vitro findings that netrin-1 promotes TCA growth and that netrin-1 blocking antibodies reduce but do not abolish the attractant effect of the ICZ on TCAs. However, because our in vitro findings suggest that netrin-1 attracts dorsal thalamic axons, it is possible that netrin-1 contributes to directing TCAs into the ICZ and that some proportion of TCAs fail to turn into the ICZ in the absence of netrin-1. An interesting issue is whether a proportion of TCAs are unable to extend through the ICZ in the absence of netrin-1, suggesting that the reduced projection would be a permanent defect, or whether it is the result of a slowing of TCA growth and given time the normal magnitude of the TCA projection would develop. Unfortunately, because the netrin-1 mutants die shortly after birth, we are not able to distinguish between these possibilities. However, either possibility is consistent with the interpretation that the ICZ is less permissive for TCA growth in the absence of netrin-1.

The reduction in the TCA projection varies between individual netrin-1 - / - mice, ranging from a 30 to $90 \%$ reduction. Such a phenotypic variation in netrin-1 $-/-$ mice has been demonstrated previously for both the optic nerve and inferior olive (IO); the optic nerve varies from a slight reduction to a near complete loss of the nerve (Deiner et al., 1997), and a variable phenotype was found in the degree of development of IO cytoarchitecture as well as cellular volume and density (Bloch-Gallego et al., 1999). Because the netrin-1 mutant is not a complete null, and a small percentage of wild-type transcripts are detected (Serafini et al., 1996), it is possible that the phenotypic variation is attributable in part to variable amounts of residual netrin-1 in the mutants. Arguing against this suggestion is the finding of a similar range of phenotypes in DCC $-/-$ mice, which are complete nulls. Bloch-Gallego et al. (1999) suggested that the phenotypic variability is caused by a "threshold" event; if more cells succeed in leaving the rhombic lips (the origin of IO cells) and reach the ventral midline (the final destination of IO cells), they may reach a critical mass that allows cellular interactions to generate rudimentary lamination. Similarly, the wide variation in the reduction of the TCA projection may be attributable to a thresholding event. In some mutants, few TCA axons initially make it to the neocortex, whereas in other mutants, more TCAs initially do. If the number of TCAs that reach the neocortex passes a certain threshold, even more TCAs might be able to "piggy back" on the initial population, thereby substantially increasing the total number that reach cortex. The difference in the number of TCAs that initially succeed in reaching the cortex might be related to potential differences in the residual amount of netrin- 1 between mutants.

In conclusion, our findings show that netrin-1 expression in the ICZ is required for the proper development of the TCA projection and that in its absence, the TCA projection through the IC is disorganized and abnormally restricted, and a reduced number of TCAs reach cortex. Our findings best support the interpretation that netrin-1 helps delineate the path of TCAs through the ventral telencephalon to the cortex by providing a growth corridor defined here as the ICZ, and that in the absence of netrin- 1 the ICZ is less permissive for TCA growth. We cannot rule out though that netrin-1 may also contribute to the turning of TCAs from the diencephalon into the ICZ. In both instances, netrin-1 does not appear to act alone, and likely is one of several attractant molecules expressed in the ICZ that control TCA growth through the ventral telencephalon. In addition to multiple molecular activities in the ICZ, other spatially and functionally distinct cues have been suggested to contribute to TCA pathfinding from dorsal thalamus to the cortex. For example, the hypothalamus releases a repellent activity for TCAs that has been suggested to promote their turning into the ICZ (Braisted et al., 1999). Furthermore, an analysis of Mash-1 mutant mice has defined a distinct cell group in the ventral telencephalon that appears to be required for TCAs to navigate from the diencephalon into the telencephalon (Tuttle et al., 1999). The normal pathfinding of TCAs likely depends on the proper deployment of each of these cues as well as others, and their selective removal should result in unique defects in the TCA projection.

\section{REFERENCES}

Blakemore C, Molnar Z (1990) Factors involved in the establishment of specific interconnections between thalamus and cerebral cortex. Cold Spring Harb Symp Quant Biol 55:491-504.

Bloch-Gallego E, Ezan F, Tessier-Lavigne M, Sotelo C (1999) Floor plate 
and netrin-1 are involved in the migration and survival of inferior olivary neurons. J Neurosci 19:4407-4420.

Braisted JE, Tuttle R, O'Leary DDM (1999) Thalamocortical axons are influenced by chemorepellent and chemoattractant activities localized to decision points along their path. Dev Biol 208:430-440.

Butler H, Juurlink BHJ (1987) An atlas for staging mammalian and chick embryos. Boca Raton: CRC.

Catalano SM, Shatz CJ (1998) Activity-dependent cortical target selection by thalamic axons. Science 281:559-562.

Catalano SM, Robertson RT, Killackey HP (1991) Early ingrowth of thalamocortical afferents to the neocortex of the prenatal rat. Proc Natl Acad Sci USA 88:2999-3003.

Catalano SM, Robertson RT, Killackey HP (1996) Individual axon morphology and thalamocortical topography in developing rat somatosensory cortex. J Comp Neurol 367:36-53.

Chenn A, Braisted JE, McConnell SK, O'Leary DDM (1997) Development of the cerebral cortex: mechanisms controlling cell fate, laminar and area patterning, and axonal connectivity. In: Molecular and cellular approaches to neural development (Cowan L, Zipursky L, Jessell T, eds.), pp 440-473. Oxford: Oxford UP.

Cohen-Tannoudji M, Babinet C, Wassef M (1994) Early determination of a mouse somatosensory cortex marker. Nature 368:460-463.

Deiner MS, Kennedy TE, Fazeli A, Serafini T, Tessier-Lavigne M, Sretavan DW (1997) Netrin-1 and DCC mediate axon guidance locally at the optic disc: loss of function leads to optic nerve hypoplasia. Neuron 19:575-589.

Friedman G, O'Leary DDM (1996) Retroviral misexpression of engrailed genes in the chick optic tectum perturbs the topographic targeting of retinal axons. J Neurosci 16:5498-5509.

Honig MG, Hume RI (1989a) Carbocyanine dyes. Novel markers for labelling neurons. Trends Neurosci 12:336-338.

Honig MG, Hume RI (1989b) Dil and diO: versatile fluorescent dyes for neuronal labelling and pathway tracing. Trends Neurosci 12:333-335 $340-331$.

Jones EG (1985) The thalamus. New York: Plenum.

Keino-Masu K, Masu M, Hinck L, Leonardo ED, Chan SS, Culotti JG, Tessier-Lavigne M (1996) Deleted in colorectal cancer (DCC) encodes a netrin receptor. Cell 87:175-185.

Kennedy TE, Serafini T, de la Torre JR, Tessier-Lavigne M (1994) Netrins are diffusible chemotropic factors for commissural axons in the embryonic spinal cord. Cell 78:425-435.

Leonardo MR, Forst S (1996) Re-examination of the role of the periplas- mic domain of EnvZ in sensing of osmolarity signals in Escherichia coli. Mol Microbiol 22:405-413.

Lumsden AG, Davies AM (1983) Earliest sensory nerve fibres are guided to peripheral targets by attractants other than nerve growth factor. Nature 306:786-788.

Metin C, Godement P (1996) The ganglionic eminence may be an intermediate target for corticofugal and thalamocortical axons. J Neurosci 16:3219-3235.

Metin C, Deleglise D, Serafini T, Kennedy TE, Tessier-Lavigne M (1997) A role for netrin-1 in the guidance of cortical efferents. Development 124:5063-5074.

Molnar Z, Blakemore C (1995) How do thalamic axons find their way to the neocortex? Trends Neurosci 18:389-397.

Molnar Z, Adams R, Blakemore C (1998) Mechanisms underlying the early establishment of thalamocortical connections in the rat. J Neurosci 18:5723-5745.

Richards LJ, Koester SE, Tuttle R, O'Leary DDM (1997) Directed growth of early cortical axons is influenced by a chemoattractant released from an intermediate target. J Neurosci 17:2445-2458.

Serafini T, Colamarino SA, Leonardo ED, Wang H, Beddington R, Skarnes WC, Tessier-Lavigne M (1996) Netrin-1 is required for commissural axon guidance in the developing vertebrate nervous system. Cell 87:1001-1014.

Serafini T, Kennedy TE, Galko MJ, Mirzayan C, Jessell TM, TessierLavigne M (1994) The netrins define a family of axon outgrowthpromoting proteins homologous to C elegans UNC-6. Cell 78:409-424.

Shirasaki R, Mirzayan C, Tessier-Lavigne M, Murakami F (1996) Guidance of circumferentially growing axons by netrin-dependent and - independent floor plate chemotropism in the vertebrate brain. Neuron 17:1079-1088.

Skarnes WC, Moss JE, Hurtley SM, Beddington RS (1995) Capturing genes encoding membrane and secreted proteins important for mouse development. Proc Natl Acad Sci USA 92:6592-6596.

Tessier-Lavigne M, Placzek M, Lumsden AG, Dodd J, Jessell TM (1988) Chemotropic guidance of developing axons in the mammalian central nervous system. Nature 336:775-778.

Tuttle R, Nakagawa Y, Johnson JE, O'Leary DDM (1999) Defects in thalamocortical axon pathfinding correlate with altered cell domains in Mash-1-deficient mice. Development 126:1903-1916.

Yee KT, Simon HH, Tessier-Lavigne M, O’Leary DDM (1999) Extension of long leading processes and neuronal migration in the mammalian brain directed by the chemoattractant netrin-1. Neuron 24:607-622. 